The INL is a

U.S. Department of Energy

National Laboratory

operated by

Battelle Energy Alliance

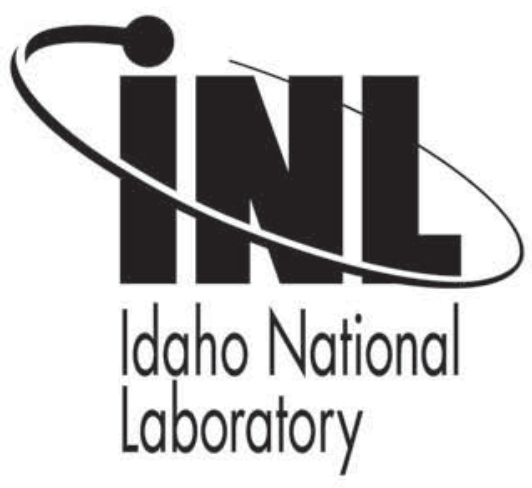

INL/CON-09-15501

PREPRINT

\title{
Eliminating
}

\section{Conservatism in the}

Piping System Analysis

Process Through

Application of a Suite of

Locally Appropriate

Seismic Input Motions

\section{ASME Pressure Vessels and Piping Division Conference}

\author{
Anthony L. Crawford \\ Robert E. Spears \\ Mark J. Russell \\ July 2009
}

This is a preprint of a paper intended for publication in a journal or proceedings. Since changes may be made before publication, this preprint should not be cited or reproduced without permission of the author. This document was prepared as an account of work sponsored by an agency of the United States Government. Neither the United States Government nor any agency thereof, or any of their employees, makes any warranty, expressed or implied, or assumes any legal liability or responsibility for any third party's use, or the results of such use, of any information, apparatus, product or process disclosed in this report, or represents that its use by such third party would not infringe privately owned rights. The views expressed in this paper are not necessarily those of the United States Government or the sponsoring agency. 


\section{ELIMINATING CONSERVATISM IN THE PIPING SYSTEM ANALYSIS PROCESS THROUGH APPLICATION OF A SUITE OF LOCALLY APPROPRIATE SEISMIC INPUT MOTIONS}

\author{
Anthony L. Crawford \\ Idaho National Laboratory \\ 2525 N. Fremont Ave \\ Idaho Falls, ID 83415 \\ Phone: (208) 526-5159 \\ Email: Anthony.Crawford@inl.gov
}

\author{
Robert E. Spears Ph.D. \\ Idaho National Laboratory \\ 2525 N. Fremont Ave \\ Idaho Falls, ID 83415 \\ Phone: (208) 526-4311 \\ Email: Robert.Spears@inl.gov
}

\author{
Mark J. Russell \\ Idaho National Laboratory \\ 2525 N. Fremont Ave \\ Idaho Falls, ID 83415 \\ Phone: (208) 526-1608 \\ Email: Mark.Russell@inl.gov
}

\begin{abstract}
Seismic analysis is of great importance in the evaluation of nuclear systems due to the heavy influence such loading has on their designs. Current Department of Energy seismic analysis techniques for a nuclear safety-related piping system typically involve application of a single conservative seismic input applied to the entire system [1]. A significant portion of this conservatism comes from the need to address the overlapping uncertainties in the seismic input and in the building response that transmits that input motion to the piping system. The approach presented in this paper addresses these two sources of uncertainty through the application of a suite of 32 earthquake realizations with equal probability of occurrence whose collective performance addresses the total uncertainty while each individual motion represents a single variation of it. It represents an extension of the soil-structure interaction analysis methodology of SEI/ASCE 43-05 [2] from the structure to individual piping components.
\end{abstract}

Because this approach is computationally intensive, automation and other measures have been developed to make such an analysis efficient. These measures are detailed in this paper.

\section{INTRODUCTION}

Seismic analysis is of great importance in the evaluation of nuclear systems due to the heavy influence such loading has on their designs. The analysis of the Idaho National Laboratory's Advanced Test Reactor Primary Coolant System, which is such a system, is the focus of this paper. Current seismic analysis techniques for a nuclear safety-related piping system involve application of a single probabilistically based seismic input applied uniformly to the entire system. Naturally, such an input requires a high level of conservatism to address uncertainty both in the seismic input and in building response.

The approach presented in this paper mitigates these two primary sources of uncertainty and establishes a more appropriate level of conservatism through the application of a suite of 32 drift corrected earthquake realizations with equal probability of occurrence. Each realization is comprised of a suite of 5 different input sources applied to the varying locally appropriate supports. Because this approach is computationally intensive, automation and other measures to make such an analysis efficient are presented and described.

Despite the attempts to reduce conservatism in the seismic input motion, limited plasticity was predicted in isolated portions of the piping system. These were handled by application of an ASME Appendix F [3] Plastic Component analysis / Elastic System Analysis approach. Such analysis can require iteration, since the elastic system loads applied to the components affect the proper elastic properties of those components in the elastic system model. This iteration is another driver for development of efficient analysis.

\section{MULTIPLE MODELS REPRESENTING PRIMARY COOLANT SYSTEM}

For this evaluation three independent piping models (Models 14, 3, and 256) are developed to represent the entire Primary Coolant System. Each of the three models is essentially decoupled from each other except for two 36 inch to 6 inch pipe connections. Due to the geometry and support 1

The United States Government retains, and by accepting the article for publication, the publisher acknowledges that the United States Government retains, a non-exclusive, paid-up, irrevocable, worldwide license to publish or reproduce the published form of this work, or allow others to do so, for United States Government purposes. 
locations the 6 inch pipe has little affect on the 36 inch pipe sections and is not included as an input into the model with the 36 inch pipe. The input to the 6 inch pipe is taken as the input motion of closest support connected to the 36 inch pipe relative to the connection. This is a result of the minimal difference between the support input motion and the resulting motion of the 36 inch to 6 inch connections.

Selecting combinations that result in independent response mitigates many of the potential disadvantages associated with a multiple model system approach to piping analysis. The primary advantage of a multiple model system for a new analysis approach is that focus can be applied to a reduced amount of the entire system and foreseen and unforeseen challenges can be identified and resolved. Fig 1 was generated to identify the model that would best serve as the pilot for this analysis by identifying how many unique components are contained in each model.

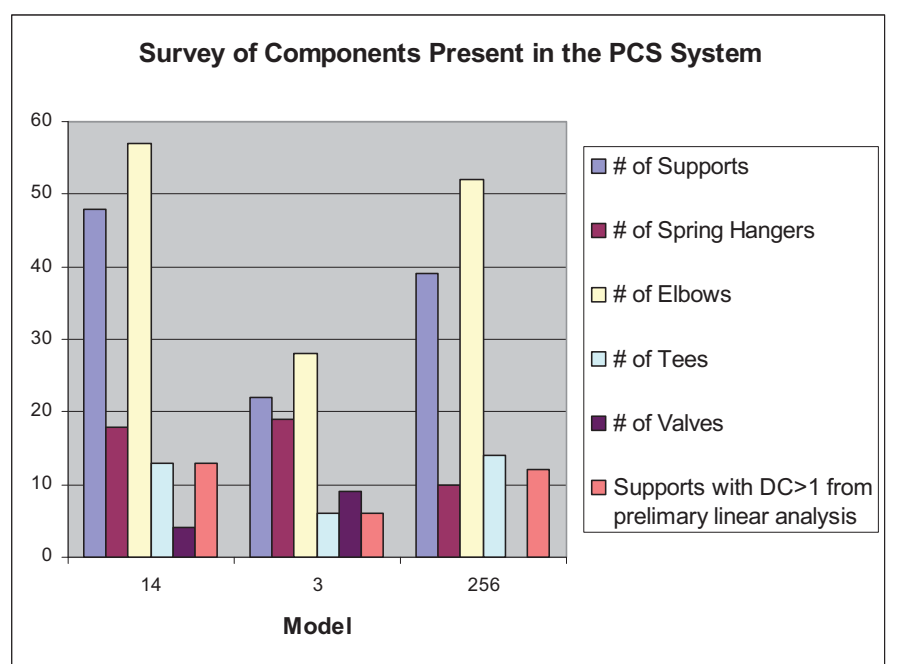

Fig 1: A survey of components present in the Primary Coolant System.

Model 3 was selected as the pilot model because each type of unique component is appropriately represented and the resulting model is still relatively simple. Model 3 will be specifically discussed in the remainder of this paper as an adequate subject to demonstrate the seismic analysis method applied to all three models.

This strategy of isolating a simply representative model was of particular value to effectively work out the technical issues pertaining to running all of the suites.

\section{FINITE ELEMENT MODEL}

The finite element models for all three models were generated using NX I-DEAS [4]. A text file was then extracted (called the input file) from the I-DEAS models to be evaluated using ABAQUS 6.7-5 [5]. The text file provided a flexible platform to efficiently run the scripts (discussed further in section 5) and incorporate the following unique items into the model:

- multiple seismic inputs applied to the appropriate groups of termination points in all three orthogonal directions

- nonlinear springs where appropriate to represent support geometry

- damping to the overall system based on its frequency response

Additional unique items that where incorporated into the finite element model prior to exporting it into the text file included:

- constant forces to represent spring hangers with dead load

- valves with the appropriate geometric properties to mimic the moment of inertia value required by the NUPIPE-II standard (3 times the bending moment of inertia values of the pipe run) [6]

- point masses to represent valve and flange masses

- material properties were adjusted to account for the temperatures and pressures associated with the system

- elbow/tee/branch (listed and unlisted) components with the appropriate moment of inertia to replicate code stiffness requirements [7]

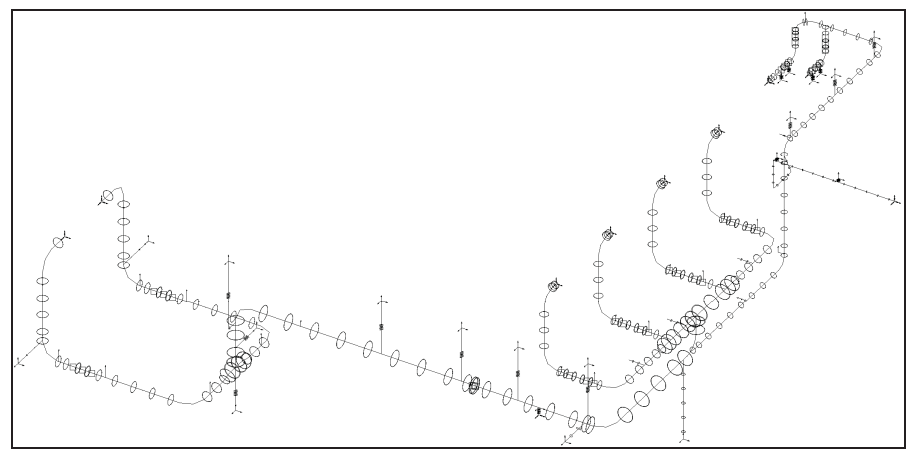

Fig 2: Finite element model of Model 3.

\subsection{MULTIPLE SEISMIC INPUTS}

The traditional approach of applying one seismic input at all termination points in a piping system generates a significant amount of unnecessary conservatism. This analysis reduced the conservatism by considering multiple seismic inputs throughout the structure encasing the Primary Coolant System. Selection of the seismic inputs applied to each termination point was primarily based on which known seismic input nodes are in the general vicinity of the termination locations and a comparison between the frequency spectrums of the local seismic input nodes.

The primary challenge associated with implementing multiple time histories was capturing the time based drift behavior of the input seismic nodes relative to one another. 2

The United States Government retains, and by accepting the article for publication, the publisher acknowledges that the United States Government retains, a non-exclusive, paid-up, irrevocable, worldwide license to publish or reproduce the published form of this work, or allow others to do so, for United States Government purposes. 
This drift behavior was not an intended feature of the time histories but was rather generated as a consequence of the numerical techniques applied in generating the inputs. Fig 3 shows the drift in the time histories for two different nodes. The large total drift does not represent a significant problem. In an evaluation, this would just cause the whole structure to translate. The relative drift (of nearly four inches for this pair), however, does represent a significant problem. This could cause significant unrealistic strains that grow with time.

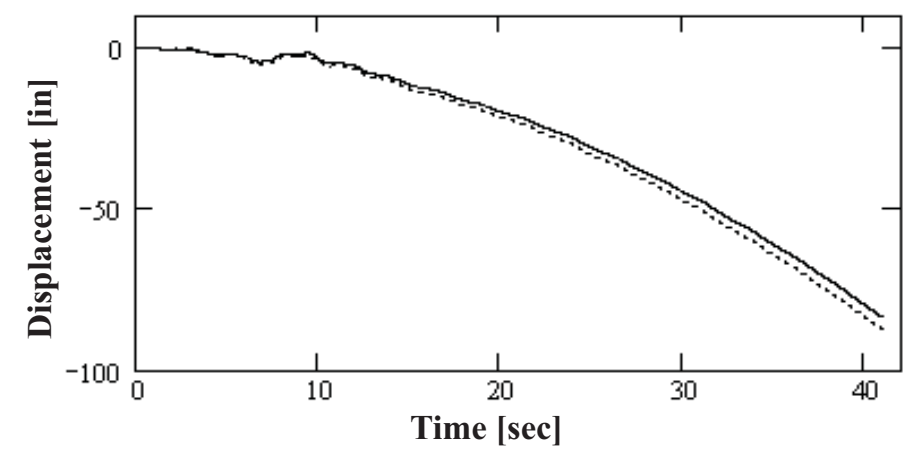

Fig 3: Drift for two nodes.

Typically drift is removed by curve fitting an acceleration polynomial to the displacement drift. The acceleration time history is then modified with the acceleration polynomial. This technique works well to remove total drift. For the given time histories in this evaluation, however, this approach does not accurately remove relative drift. To more accurately remove the relative (and total) drift, initial acceleration and velocity constants are established for each displacement time history that corrects the global response. This is performed with a least squares fit.

Acceleration time histories are the time histories used for evaluation. Each displacement time history is found by integrating its corresponding acceleration time history twice. Consequently, the drift correction is performed on the acceleration time history. The constant acceleration correction is easily incorporated by subtracting it from the entire acceleration time history. Fig 4 shows the modification to the drift for the two nodes resulting from this step.

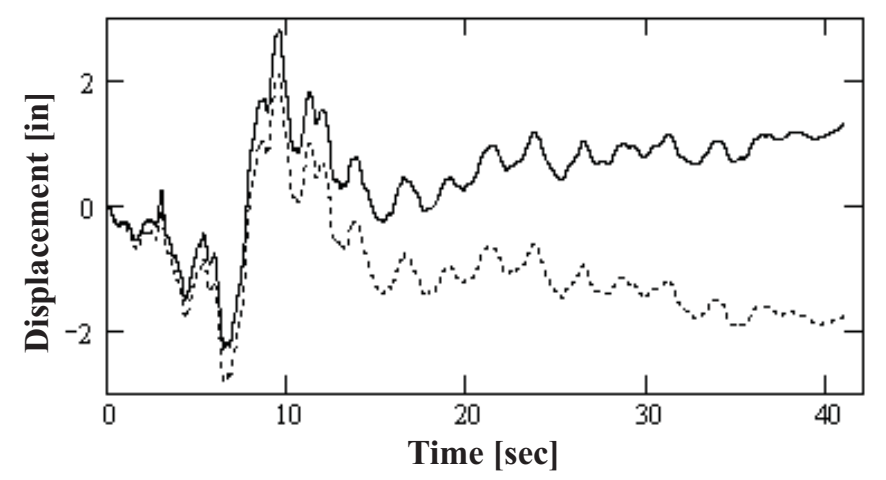

Fig 4: Acceleration corrected drift for two nodes.
Addressing the initial velocity is more difficult as the modifications are performed on an acceleration time history. This correction is accomplished with a smooth polynomial acceleration time history that modifies the start of the acceleration time history. The time duration is short to avoid affecting the strong motion of the acceleration time history. It must include enough time history points to produce an accurate response, however. Fig 5 shows the polynomial used to correct the displacement time history (solid curve) in Fig 3.

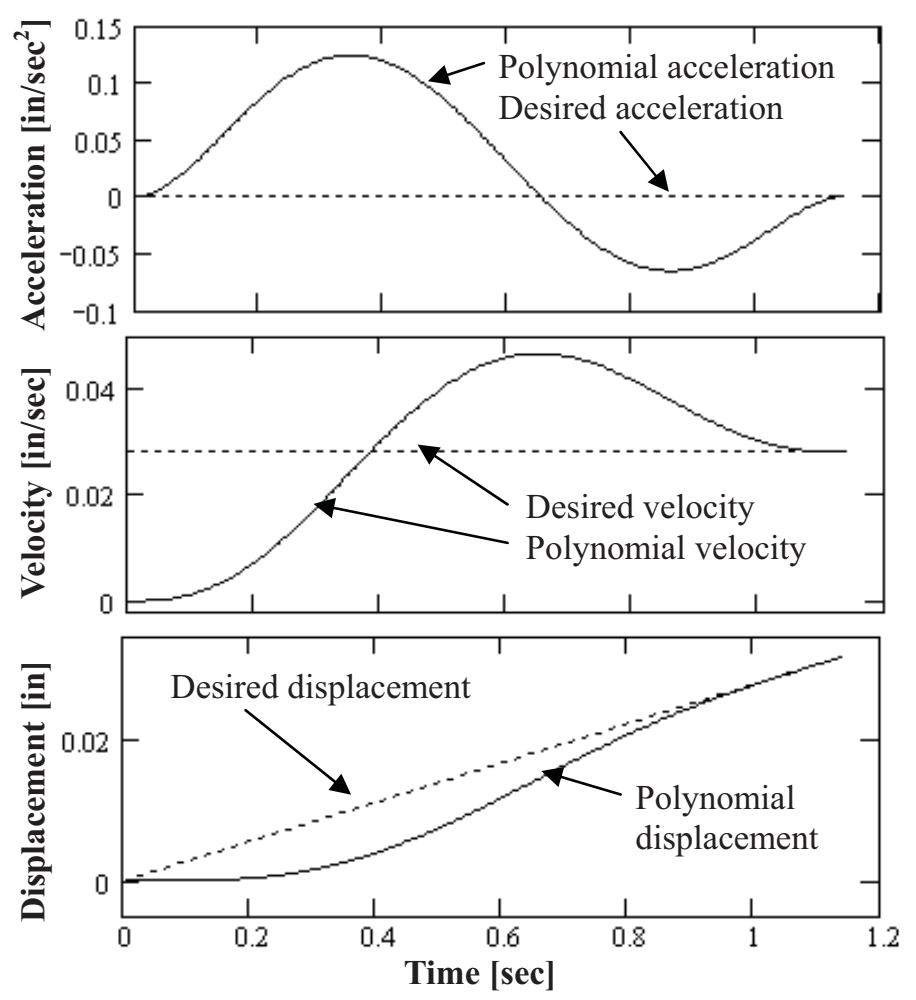

Fig 5: Smooth polynomial motion correction.

Performing the velocity correction on the two displacement time histories in Fig 4 produces the indistinguishable curves shown in Fig 6. Fig 7 shows the difference in the two drift corrected displacement time histories.

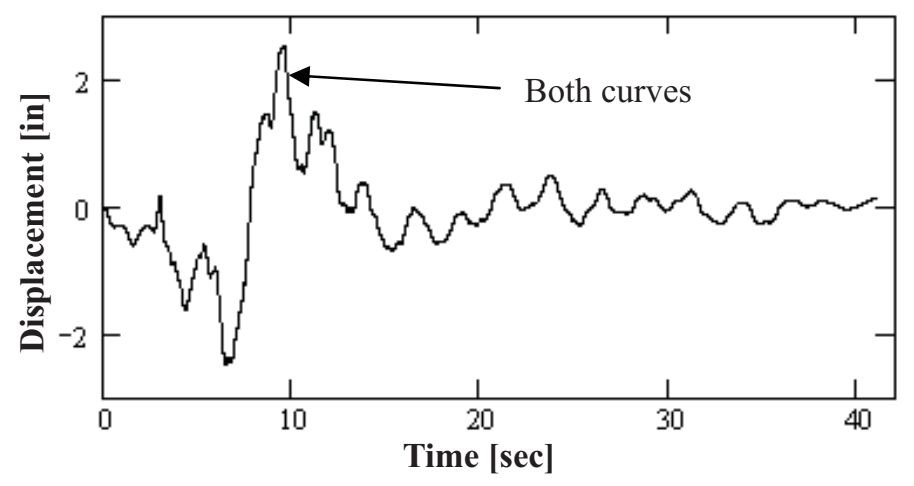

Fig 6: Velocity corrected drift for two nodes.

The United States Government retains, and by accepting the article for publication, the publisher acknowledges that the United States Government retains, a non-exclusive, paid-up, irrevocable, worldwide license to publish or reproduce the published form of this work, or allow others to do so, for United States Government purposes. 


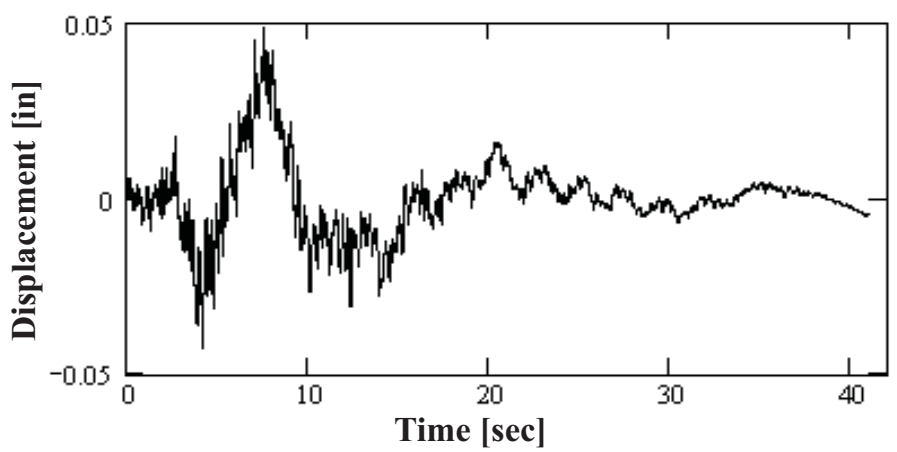

Fig 7: Relative corrected drift for two nodes.

The time history in Fig 7 represents a reasonable relative motion between two nodes in the model. Also, evaluating acceleration response spectra before and after this drift correction causes an insignificant change (for the time histories used in this evaluation). Fig 8 shows the before and after drift correction acceleration response spectra for one node. The maximum difference in the two curves is less than $0.2 \mathrm{in} / \mathrm{sec}^{2}$.

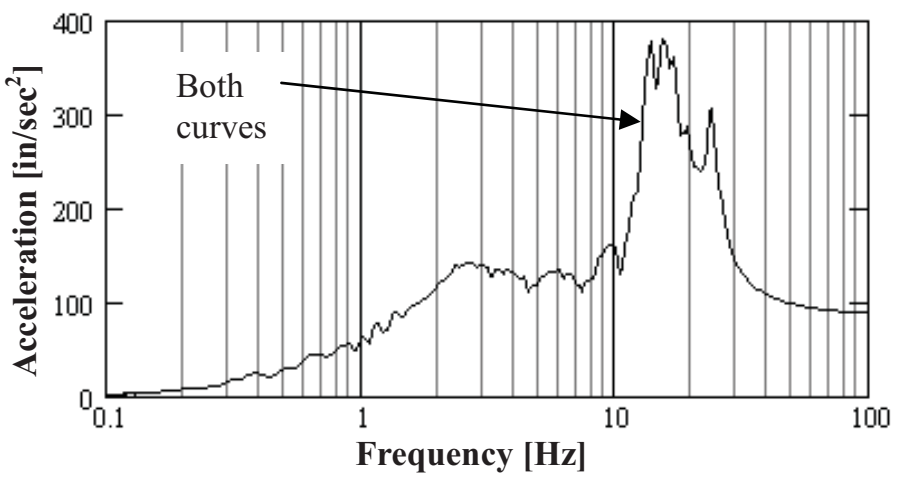

Fig 8: Before and after correction response spectra.

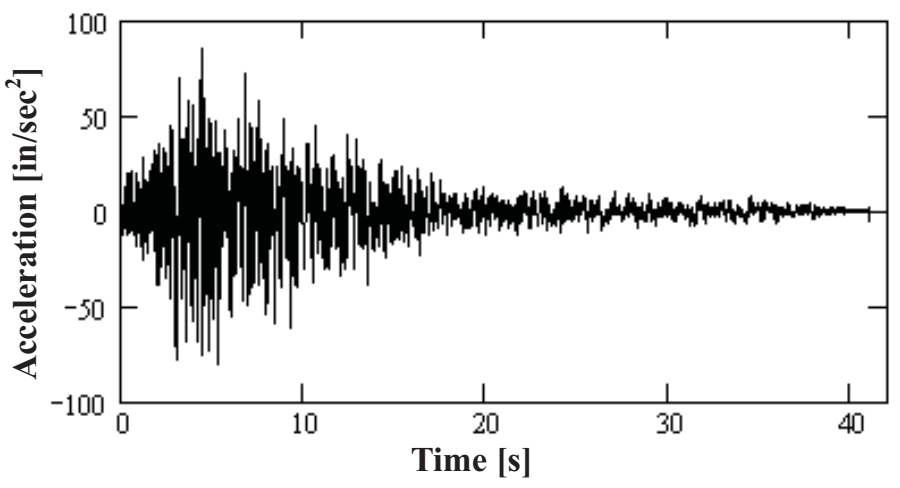

Fig 9: Before and after acceleration time histories.
Direct time integration in ABAQUS is performed with Rayleigh damping as calculated by equation 1 .

$$
\zeta=\frac{\alpha}{2 \cdot \omega}+\frac{\beta \cdot \omega}{2}
$$

In equation 1 the damping value " $\zeta$ " is a function of natural frequency " $\omega$ " and the Rayleigh damping coefficients " $\alpha$ " and " $\beta$ ". The Rayleigh damping coefficients were calibrated to minimize the error compared to modal damping while preserving a conservative bias.

Fig 10 - Fig 14 show how the Rayleigh damping coefficients are calibrated. These figures represent some of the data used to establish the Rayleigh damping coefficients for Model 3. Fig 10 shows the cumulative effective mass in the three translational directions for Model 3. The "H1" direction is east-west, the "H2" direction is north-south, and the "V" direction is vertical. Considering Fig 10, the first significant response in any direction occurs around $1.8 \mathrm{~Hz}$. (A significant response appears on this plot as a "steep" region or sudden "jump" in a given curve.) Between $1.8 \mathrm{~Hz}$ and $50 \mathrm{~Hz}$ are many frequencies where significant response occurs.

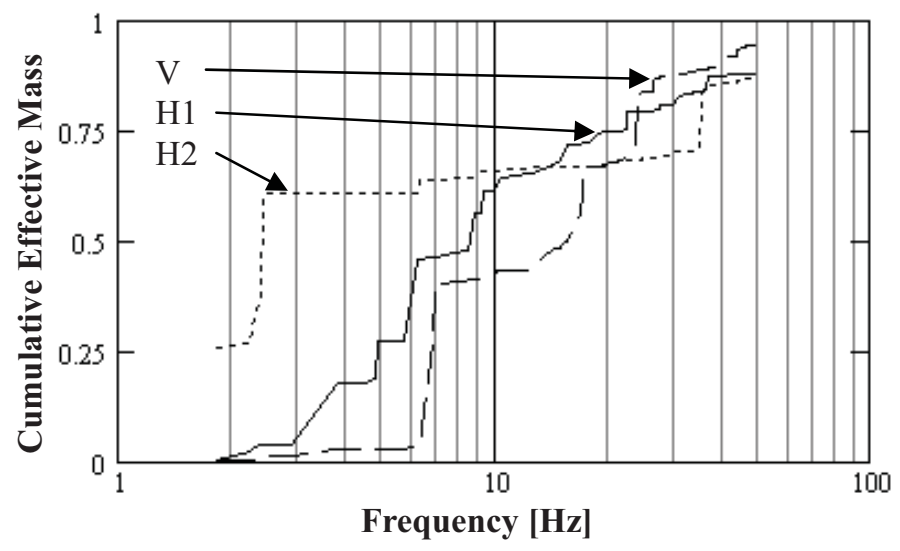

Fig 10: Cumulative effective mass ratio.

Fig 11 shows the 5\% damped response spectra data for all 32 time histories for the "H1" direction. An $80^{\text {th }}$ percentile curve is selected with effective mass weighting. The $80^{\text {th }}$ percentile was chosen as it is currently being the item being incorporated into the ASCE 4-98 code. At each effective mass output frequency, the effective mass is multiplied by the response at that location for a given response spectrum. For each response spectrum, these values are summed over the entire range of effective mass values. The 32 response spectra are then ordered based on this summed value and the $80^{\text {th }}$ percentile curve is identified.

\subsection{DAMPING}

A modal damping value of $5 \%$ is applicable to this evaluation, as listed in Table 3-2 of ASCE/SEI 43-05 [4]. To accommodate support nonlinearity (gaps and lift-off), the finite element model runs were performed using direct time integration.

The United States Government retains, and by accepting the article for publication, the publisher acknowledges that the United States Government retains, a non-exclusive, paid-up, irrevocable, worldwide license to publish or reproduce the published form of this work, or allow others to do so, for United States Government purposes. 


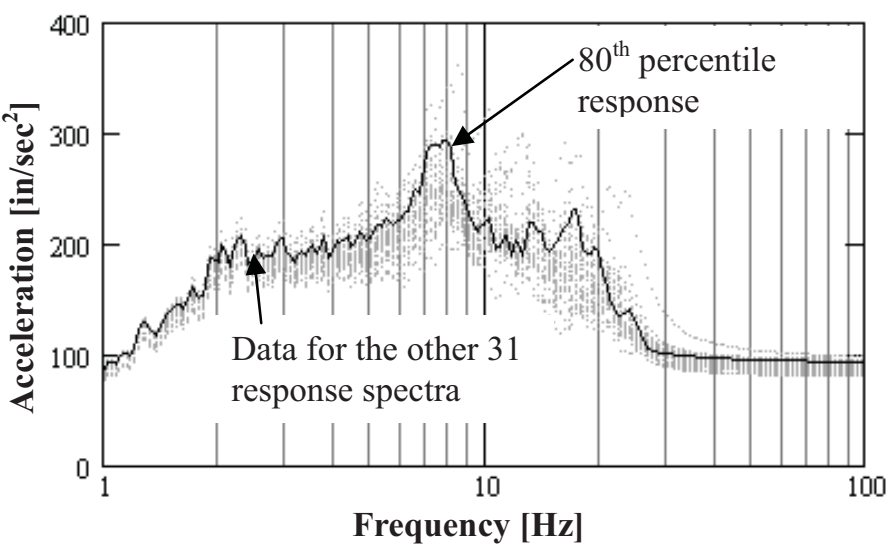

Fig 11: H1 response spectra for 5\% damping.

Fig 12 shows the Rayleigh damping curve that is established by an iterative process including response in all three directions. The low frequency location where the Rayleigh damping curve crosses 5\% damping is based on the $1.8 \mathrm{~Hz}$ value for the first significant response in any direction. The higher frequency crossing is found by an iterative process. The final iteration results are shown in Fig 12 considering a higher frequency $5 \%$ crossing of $9.4 \mathrm{~Hz}$.

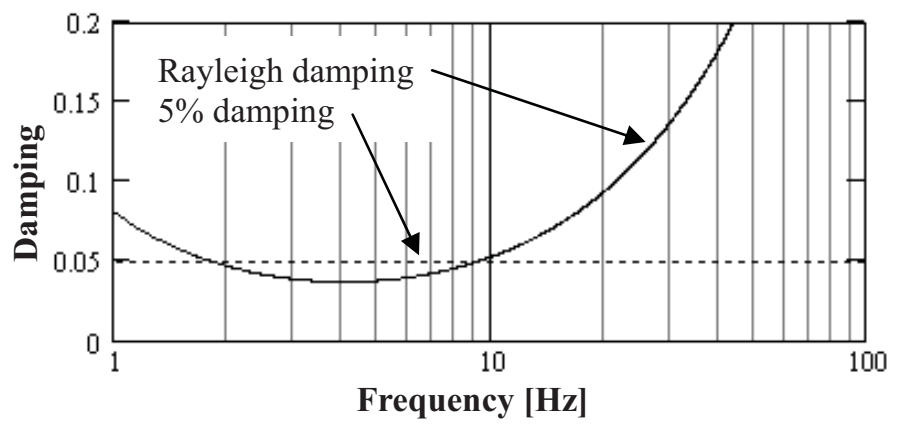

Fig 12: Rayleigh damping versus frequency.

The Rayleigh damping curve shown in Fig 12 is justified using the following process. First, the Rayleigh damped response is calculated for the $80^{\text {th }}$ percentile acceleration time history (as shown in Fig 13).

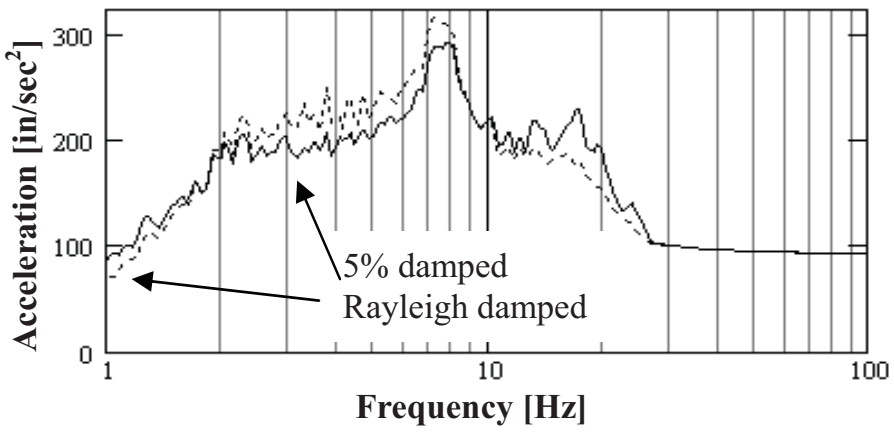

Fig 13: H1 Rayleigh and 5\% damped response spectra.
Next, at each effective mass output frequency, the effective mass is multiplied by the Rayleigh damped response minus the $5 \%$ damped response at that frequency. Fig 14 shows how these values sum as a function of frequency. Summing all these values for all three response directions produces a single value (shown as the last value in Fig 14). If this value is positive, then the Rayleigh damping curve is considered acceptable.

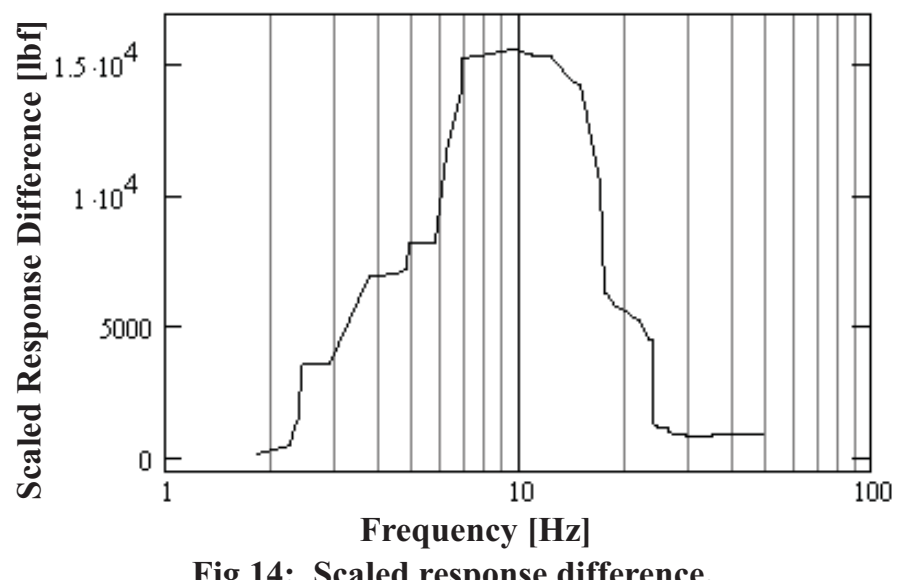

Fig 14 is plotted as an indication of the significance of the last value. As can be seen in the curve, the last value is significantly positive, but it is not large compared to the peak values. Using this Rayleigh damping curve, the average response of the given piping model should to be greater than that of the same piping model with 5\% modal damping.

\section{POST PROCESSING}

After incorporating the nonlinear springs, multiple seismic inputs, and damping into the text file it was processed using ABAQUS 6.7-5 and the element/nodal results where extracted. The results where then post processed to identify the maximum Demand to Capacity ratio (D/C) of every element for each of the 32 realizations. The complete sets of results were then combined, sorted, and the $80^{\text {th }}$ percentile $\mathrm{D} / \mathrm{C}$ value was identified for every component. The process of identifying the $80^{\text {th }}$ percentile $\mathrm{D} / \mathrm{C}$ value is currently being incorporated in reference [1].

The above goal was achieved with the use of the Mathcad software and its loop logic to iterate through all of the results but other software packages are certainly capable of performing the calculations. While performing the calculations it was very beneficial to carry additional information in a vector form as the loops iterated through the data. The additional information carried included the time that the maximum $\mathrm{D} / \mathrm{C}$ value occurred, the forces/moments experienced at that time, and the node or element that it occurred at.

The United States Government retains, and by accepting the article for publication, the publisher acknowledges that the United States Government retains, a non-exclusive, paid-up, irrevocable, worldwide license to publish or reproduce the published form of this work, or allow others to do so, for United States Government purposes. 


\section{SCRIPTING TO EFFICIENTLY EXECUTE ANALYSIS}

Scripts were crucial in order to process the enormous amount of data relatively quickly and consistently. Prior to implementation of the scripts, a general text file that possessed all of the consistent model information between each realization was required. This general file was generated by taking the I-DEAS model text file and incorporating the appropriate damping and nonlinear springs. The scripts subjected the generated text file to the following sequence of tasks to perform this evaluation:

- generate 32 separate input files, each with the appropriate seismic input node time histories

- run each of the 32 generated input files through ABAQUS to generate the desired element/nodal force output

- run each of the 32 element/nodal force outputs through ABAQUS Viewer to extract the element/nodal force output into a desired format

- run each Mathcad worksheet to calculate the maximum $\mathrm{D} / \mathrm{C}$ values of every component for each of the 32 realizations

\section{CONCLUSIONS}

This paper presented an effective seismic analysis process capable of reducing the conservatism inherent in current seismic analyses methods. Techniques for addressing aspects typically neglected in other analyses such as elbow/tee/branch stiffness correction, multiple time histories, model damping, and the post processing where discussed. This analysis method proved to be very effective in accurately modeling and analyzing the system as it was subjected to the multiple seismic events.

\section{ACKNOWLEDGMENTS}

This manuscript has been authored by Battelle Energy Alliance, LLC under Contract No. DE-AC07-05ID14517 with the U.S. Department of Energy. The United States Government retains and the publisher, by accepting the article for publication, acknowledges that the United States Government retains a nonexclusive, paid-up, irrevocable, world-wide license to publish or reproduce the published form of this manuscript, or allow others to do so, for United States Government purposes.

The work described in this paper was performed as part of the Advanced Test Reactor Life Extension Project.

\section{REFERENCES}

1. ASCE 4-98 Seismic Analysis of Safety-Related Nuclear Structes and Commentary, American Society of Civil Engineers, 1998.

2. ASCE/SEI 43-05 Seismic Design Criteria for Structure, Systems, and Components in Nuclear Facilities, American Society of Civil Engineers, 2005.
3. ASME Boiler and Pressure Vessel Code, Section III, Division 1, Appendix F, Rules for Evaluation of Service Loadings with Level D Service Limits, American Society of Mechanical Engineers, July 2007.

4. I-DEAS Simulation, Electronic Data Systems, Maryland Heights, MO.

5. ABAQUS Inc., ABAQUS Standard, Version 6.7-5, Rising Sun Mills, 166 Valley Street, Providence, RI, 02909-2499, Tel: +1-401-276-4400, email: support@Abaqus.com.

6. Quadrex Corporation, "NUPIPE-II Piping Analysis Program With Snuber Optimization, Program Description and User's manual," Version 1.8.1, Revision 0.

7. 2007 ASME Boiler and Pressure Vessel Code, Section III, "Rules for Construction of Nuclear Facility Components," Division 1, Subsection NB, "Class 1 Components," American Society of Mechanical Engineers, July 2007.

The United States Government retains, and by accepting the article for publication, the publisher acknowledges that the United States Government retains, a non-exclusive, paid-up, irrevocable, worldwide license to publish or reproduce the published form of this work, or allow others to do so, for United States Government purposes. 